\title{
IncRNA FAM99A is downregulated in preeclampsia and exerts a regulatory effect on trophoblast cell invasion, migration and apoptosis
}

\author{
TONGQIANG HE ${ }^{1}$, YUAN QIAO ${ }^{1}$, YANXIANG LV ${ }^{1}$, JUN WANG ${ }^{1}$, RUI HU ${ }^{1}$ and YINLI CAO ${ }^{2}$ \\ ${ }^{1}$ Obstetrics and Gynecology Intensive Care Unit; ${ }^{2}$ Department of Obstetrics, \\ The Northwest Women and Children's Hospital, Xi'an, Shaanxi 718900, P.R. China
}

Received April 19,2018; Accepted May 3, 2019

DOI: $10.3892 / \mathrm{mmr} .2019 .10350$

\begin{abstract}
Preeclampsia (PE) is a complication of pregnancy, and a leading cause of maternal mortality and morbidity worldwide. Recently, the dysregulation of long non-coding RNAs (IncRNAs) has been reported to contribute to the pathogenesis and progression of PE. This study aimed to examine the alterations in the IncRNA family with sequence similarity 99 member A (FAM99A) in PE and its effects on trophoblasts. The results of reverse transcription-quantitative PCR indicated that the expression levels of FAM99A were downregulated in placental tissues from women with severe PE compared with in those from controls. A Transwell invasion assay and wound healing assay revealed that overexpression of FAM99A promoted invasion and migration of HTR-8/SVneo cells; conversely, knockdown of FAM99A suppressed the invasive and migratory abilities of HTR-8/SVneo cells. Flow cytometry demonstrated that FAM99A overexpression induced a decrease in the apoptotic rate of cells, whereas knockdown of FAM99A increased the apoptotic rate of HTR-8/SVneo cells. Western blot analysis revealed that overexpression of FAM99A decreased the protein expression levels of cleaved caspase-3, cleaved caspase- 9 and Bax, and increased $\mathrm{Bcl}-2$ protein expression, whereas knockdown of FAM99A had the opposite effects on these protein levels. Overexpression of FAM99A also decreased caspase-3 activity in HTR-8/SVneo cells; however, knockdown of FAM99A increased caspase-3 activity. In addition, overexpression of FAM99A enhanced Wnt/ $\beta$-catenin signaling activity, whereas FAM99A knockdown exerted an inhibitory effect on the $\mathrm{Wnt} / \beta$-catenin signaling activity in HTR-8/SVneo cells. In conclusion, these results indicated
\end{abstract}

Correspondence to: Dr Yinli Cao, Department of Obstetrics, The Northwest Women and Children's Hospital, 1616 Yanxiang Road, Xi'an, Shaanxi 718900, P.R. China

E-mail: xbfnetyy029@163.com

Key words: preeclampsia, family with sequence similarity 99, trophoblasts, invasion and migration, apoptosis, Wnt/ $\beta$-catenin that FAM99A may serve a role in modulating the functions of trophoblasts, partially via targeting $\mathrm{Wnt} / \beta$-catenin signaling.

\section{Introduction}

Preeclampsia (PE) is a complication of pregnancy, which is characterized by proteinuria and hypertension after 20 weeks of gestation. It remains a leading cause of maternal mortality and morbidity worldwide (1). Although great advances have been made in this field, the precise mechanisms underlying PE remain unclear. It is generally believed that the placenta serves an essential role in PE pathogenesis and progression (2). During early development of the placenta, the uterine spiral arteries of the decidua and the myometrium are initially invaded by extravillous trophoblasts (EVTs) of fetal origin; the invaded EVTs subsequently replace the endothelial layers of the maternal spiral arteries $(3,4)$. This process causes an increase in utero-placental perfusion and a decrease in maternal blood flow resistance. In PE, this process is impaired, which may be due to increased apoptosis, decreased proliferation of EVTs, and abnormal migration and invasion of EVTs (5). A previous study reported that apoptosis of placental villous trophoblasts in PE pregnancies is increased due to hypoxia-reperfusion injury and oxidative stress (6). Although it has been suggested that increased apoptosis may promote syncytial degeneration and release inflammatory mediators into the maternal circulation, the role of increased apoptosis in the pathogenesis of PE remains unclear (7). Therefore, understanding the molecular mechanisms underlying the abnormal functions of trophoblast cells may assist in improving the management of PE.

Long non-coding RNAs (lncRNAs) are defined as a type of non-coding RNA > 200 nucleotides long. Previous studies have demonstrated roles for IncRNAs in the pathogenesis and progression of diseases, including cancer, neurodegenerative diseases and cardiovascular diseases (8-10). Several lncRNAs have been identified to serve biological roles in PE. The lncRNA maternally expressed gene 3 (MEG3) is downregulated in placental tissues from patients with $\mathrm{PE}$, and is associated with trophoblast cell apoptosis and migration (11). The lncRNA metastasis-associated lung adenocarcinoma transcript 1 (MALAT1) has also been identified to be downregulated in PE and to regulate JEG-3 trophoblast cellular 
processes, including cell proliferation, cell apoptosis, cell migration and invasion (12). The lncRNA SPRY4 intronic transcript 1 (SPRY4-IT1) regulates the epithelial-mesenchymal transition, and modulates trophoblast cell invasion and migration (13). Recently, data from microarray analysis revealed that the lncRNA family with sequence similarity 99 member A (FAM99A) is downregulated in PE (14), suggesting a potential role for FAM99A in PE. However, the biological role of FAM99A in PE remains unknown.

The present study examined the expression levels of FAM99A in placental tissues from women with PE, and determined the in vitro effects of FAM99A on trophoblast cell invasion, migration and apoptosis. The present results demonstrated that FAM99A may provide a potential novel approach for the diagnosis and treatment of PE.

\section{Materials and methods}

Clinical samples. A total of 45 healthy pregnant women and 45 women with severe $P E$ (early onset, $n=24$; late onset, $n=21$ ) were included in the present study. Placental tissues were collected from primipara women who underwent a caesarean section between January 2015 and June 2017 at The Northwest Women and Children's Hospital. All of the collected placental tissues were washed with sterile PBS and immediately placed in liquid $\mathrm{N}_{2}$; the sections were stored at $-80^{\circ} \mathrm{C}$ for further experimentation. All experiments were approved by the Ethics Committee of The Northwest Women and Children's Hospital, and written informed consent was obtained from all of the recruited subjects. All clinical studies were performed according to the principles of the Declaration of Helsinki. None of the patients had autoimmune diseases, chronic nephritis, diabetes, heart diseases, chronic hypertension, thrombophilic conditions or HELLP syndrome (hemolysis, elevated liver enzymes and low platelet count), and none delivered vaginally or gave birth to infants with fetal malformation. Severe PE was diagnosed based on the definition provided in Williams Obstetrics (23rd edition) (15). The patients had no history of preexisting or chronic hypertension, but had exhibited systolic blood pressure $>160 \mathrm{mmHg}$ or diastolic blood pressure $>110 \mathrm{mmHg}$ on at least two occasions, accompanied by significant proteinuria $(>300 \mathrm{mg} / 24 \mathrm{~h}$ ), or persistent and severe central nervous system symptoms, or symptoms in multiple organs associated with persistent epigastric or right upper-quadrant pain, after the 20th week of gestation. Early onset of PE is defined as PE that develops prior to 34 weeks of gestation, whereas late onset of PE develops at $\geq 34$ weeks of gestation (16). The key clinical characteristics of the recruited subjects are summarized in Table I.

Cell culture. HTR-8/SVneo cells (CRL-3271 ${ }^{\mathrm{TM}}$ ) were purchased from American Type Culture Collection and were authenticated by short tandem repeat profiling (17). HTR-8/SVneo cells were maintained in RPMI 1640 medium supplemented with $10 \%$ fetal bovine serum (FBS; Gibco; Thermo Fisher Scientific, Inc.), $100 \mu \mathrm{g} / \mathrm{ml}$ streptomycin and $100 \mathrm{U} / \mathrm{ml}$ penicillin at $37^{\circ} \mathrm{C}$ in a humidified incubator containing $5 \% \mathrm{CO}_{2}$.

Transfection with plasmids and small interfering RNAs (siRNAs). The control plasmid (pcDNA3.1) and the FAM99A overexpressing plasmid (pcDNA3.1-FAM99A) were commercially designed and synthesized by Shanghai GenePharma Co., Ltd. Scrambled siRNA (siRNA-negative control, si-NC) and FAM99A-specific siRNA (si-FAM99A) were commercially synthesized by Guangzhou RiboBio Co., Ltd. Cell transfection ( $1 \times 10^{6}$ cells $\left./ \mathrm{ml}\right)$ with plasmids $(2 \mu \mathrm{g})$ or siRNAs $(50 \mathrm{nM})$ was performed at room temperature using Lipofectamine ${ }^{\circledR} 2000$ reagent (Invitrogen; Thermo Fisher Scientific, Inc.) according to the manufacturer's protocol. A total of $48 \mathrm{~h}$ post-transfection, cells were used for further experimentation.

$R N A$ extraction and reverse transcription-quantitative $P C R$ $(R T-q P C R)$. Total RNA was isolated from placental tissues or cells using TRIzol ${ }^{\circledR}$ reagent (Invitrogen; Thermo Fisher Scientific, Inc.) according to the manufacturer's protocol. RNA was reversed transcribed into cDNA using a Reverse Transcription kit (Takara Biotechnology Co., Ltd.) according to the manufacturer's protocol. SYBR Premix Ex Taq (Takara Biotechnology Co., Ltd.) was used to perform qPCR in order to determine the expression levels of FAM99A, according to the manufacturer's protocol. The primers were as follow: FAM99A; forward, 5'-GTCCCTTGCCCTCTCTTGTC-3' and reverse, 5'-ACACGCATCACAAAACAGCC-3'; and GAPDH; forward, 5'-GTCAACGGATTTGGTCTGTATT-3' and reverse, 5'-AGTCTTCTGGGTGGCAGTGAT-3'. The thermocycling conditions were as follows: $95^{\circ} \mathrm{C}$ for $30 \mathrm{sec}$, followed by 40 cycles at $95^{\circ} \mathrm{C}$ for $5 \mathrm{sec}$ and $60^{\circ} \mathrm{C}$ for $30 \mathrm{sec}$. Gene expression levels were normalized to GAPDH. RT-qPCR assays were performed on an ABI 7500 system (Applied Biosystems; Thermo Fisher Scientific, Inc.). The gene expression levels were calculated using the comparative $\mathrm{Ct}$ method (18).

Transwell invasion assay. The invasive ability of HTR-8/SVneo cells was assessed using a Transwell invasion assay with Matrigel. Briefly, transfected HTR-8/SVneo cells $\left(1 \times 10^{6}\right.$ cells $\left./ \mathrm{ml}\right)$ were re-suspended in $200 \mu \mathrm{l}$ serum-free medium and cultured in the upper chambers of Matrigel-coated (Corning, Inc.) Transwell inserts ( $8 \mu \mathrm{m}$ pore size; Corning Inc.). The lower chamber was filled with $800 \mu$ l culture medium supplemented with $10 \%$ FBS. After culturing for $24 \mathrm{~h}$, the cells on the top surface were removed and the cells on the bottom surface were fixed with $70 \%$ ethanol for $10 \mathrm{~min}$ at room temperature and stained with $0.1 \%$ crystal violet for $10 \mathrm{~min}$ at room temperature. The invaded cells were counted under a light microscope.

Wound healing assay. The migratory ability of HTR-8/SVneo cells was assessed using a wound healing assay. Briefly, the transfected HTR-8/SVneo cells $\left(1 \times 10^{6}\right.$ cells $\left./ \mathrm{ml}\right)$ were seeded in a 6-well plate and were grown until they reached $89-90 \%$ confluence. Wounds were generated by scratching the cell layers with a 1-ml pipette tip and cell proliferation was blocked with $40 \mu \mathrm{M}$ mitomycin $\mathrm{C}$ (Sigma-Aldrich; Merck KGaA). Cells were incubated for another $24 \mathrm{~h}$ at $37^{\circ} \mathrm{C}$. Wound width was measured using a light microscope at 0 and $24 \mathrm{~h}$ after the wound was created, and the percentage of wound closure was calculated.

Flow cytometry. Cell apoptosis was detected using a fluorescein isothiocyanate (FITC) Annexin V Apoptosis Detection 
Table I. Clinical characteristics of normal and PE pregnancies.

$\operatorname{PE}(\mathrm{n}=45)$

\begin{tabular}{lccc}
\cline { 2 - 3 } Characteristics & Control $(\mathrm{n}=45)$ & Early onset $(\mathrm{n}=22)$ & Late onset $(\mathrm{n}=23)$ \\
\hline Maternal weight $(\mathrm{kg})$ & $68.9 \pm 5.6$ & $70.8 \pm 6.7$ & $71.4 \pm 6.0$ \\
Maternal age (years) & $30.6 \pm 4.3$ & $30.8 \pm 3.6$ & $30.0 \pm 3.8$ \\
Gestational age (weeks) & $38.7 \pm 2.3$ & $37.2 \pm 1.8$ & $37.9 \pm 2.1$ \\
Systolic blood pressure (mmHg) & $113.2 \pm 9.8$ & $166.2 \pm 5.9^{\mathrm{a}}$ & $170.6 \pm 6.8^{\mathrm{a}}$ \\
Diastolic blood pressure (mmHg) & $75.3 \pm 3.4$ & $108.2 \pm 7.2^{\mathrm{a}}$ & $108.6 \pm 6.5^{\mathrm{a}}$ \\
Proteinuria (g/day) & Not detected & $4.4 \pm 1.4^{\mathrm{a}}$ & $4.6 \pm 1.1^{\mathrm{a}}$ \\
Body weight of infant (g) & $3,556.5 \pm 346.1$ & $2,524.8 \pm 146.5^{\mathrm{a}}$ & $2,560.8 \pm 184.7^{\mathrm{a}}$ \\
\hline
\end{tabular}

${ }^{\mathrm{a}} \mathrm{P}<0.05$ vs. control. $\mathrm{PE}$, preeclampsia.

kit (Sigma-Aldrich; Merck KGaA). Transfected HTR-8/SVneo cells $\left(1 \times 10^{7}\right.$ cells $\left./ \mathrm{ml}\right)$ were collected using trypsin and double-stained with propidium iodide and FITC-Annexin V, according to the manufacturer's protocol. Cell apoptosis was analyzed by flow cytometry (FACScan; BD Biosciences) and apoptotic rate was calculated using CellQuest analysis software (version 5.1; BD Biosciences).

Caspase-3 activity. The caspase-3 activity of HTR-8/SVneo cells was measured using a Caspase-3 assay kit (cat.no. CASP3C-1KT; Sigma-Aldrich; Merck KGaA) according to the manufacturer's protocol. Briefly, the transfected cells were collected and lysed with the lysis buffer supplied with the kit. The supernatant of the lysed cells was collected by centrifugation at 2,000 x $\mathrm{g}$ for 15 min and the supernatant was incubated with Ac-DEVE-pNA and the reaction buffer. Caspase- 3 activity was determined by measuring optical density at $405 \mathrm{~nm}$.

TOP-FLASH luciferase assay. Cells $\left(1 \times 10^{6}\right.$ cells $\left./ \mathrm{ml}\right)$ were transfected with the TOP-FLASH reporter construct $(0.5 \mu \mathrm{g})$ together with the Renilla luciferase vector $(0.05 \mu \mathrm{g})$ at room temperature for $48 \mathrm{~h}$ using Lipofectamine 2000 reagent (Invitrogen; Thermo Fisher Scientific, Inc.). A total of $48 \mathrm{~h}$ post-transfection, luciferase activity was measured using the Dual Luciferase Reporter Assay system (Promega Corporation) according to the manufacturer's protocol. Renilla luciferase activity was used as an internal control to normalize the TOP values.

Western blot analysis. Cells were lysed with RIPA lysis buffer (Beyotime Institute of Biotechnology) for total protein extraction. Protein concentrations were measured by BCA protein assay (Bio-Rad Laboratories, Inc., Hercules, USA) and equal amounts of proteins $(50 \mu \mathrm{g})$ were separated by $10 \%$ sodium dodecyl sulfate-polyacrylamide gel electrophoresis and transferred onto a polyvinylidene difluoride (PVDF) membrane. The PVDF membrane was incubated with $5 \%$ non-fat milk for $1 \mathrm{~h}$ at room temperature, followed by incubation with corresponding primary antibodies against cleaved caspase-3 (1:1,000; cat. no. ab2302; Abcam, Cambridge, UK), cleaved caspase-9 (1:1,000; cat. no. ab2324; Abcam), Bcl-2 (1:1,000; cat. no. ab32124; Abcam), BAX
(1:1,500; cat. no. ab32503; Abcam), $\beta$-catenin $(1: 2,000$; cat. no. ab16051; Abcam), GSK-3 $\beta$ (1:500; cat. no. ab131356; Abcam), DKK-1 (1:2,000; cat. no. ab109416; Abcam), c-myc (1:1,000; cat. no. ab39688; Abcam) and $\beta$-actin $(1: 2,000$; cat. no. ab8229; Abcam) overnight at $4^{\circ} \mathrm{C}$. The membrane was then washed three times with PBS, followed by incubation with horseradish peroxidase-conjugated secondary antibody (1:5,000; cat. no. ab205718; Abcam) at room temperature for $1 \mathrm{~h}$. The western blot bands were detected using an enhanced chemiluminescence kit (Abcam) and protein levels were semi-quantified using Image $\mathbf{J}$ software (version 1.8.0; National Institutes of Health, Bethesda, MD, USA).

Statistical analysis. All data are presented as the mean \pm standard deviation of three experimental repeats. Data analysis was performed using GraphPad Prism (version 6.0; GraphPad Software, Inc.). Student's t-test or one-way ANOVA followed by Bonferroni's multiple comparison test were used to evaluate significant differences between groups. $\mathrm{P}<0.05$ was considered to indicate a statistically significant difference.

\section{Results}

Clinical characteristics and FAM99A expression in placental tissues. Clinical data were collected from all participants. The patients were divided into two groups: Normal pregnancy $(n=45)$ and PE pregnancy $(n=45)$. As shown in Table I, the gestational age in the early and late onset PE groups was lower than that in the control group, and the body weight of infants was lower in the early and late onset PE group compared with the control group. Furthermore, the patients with early or late onset PE had higher systolic and diastolic blood pressure compared with the control group, and exhibited proteinuria.

RT-qPCR was performed to determine the expression levels of FAM99A in placental tissues from healthy women and women with early onset $(n=22)$ and late onset $(n=23)$ PE. The expression levels of FAM99A were significantly decreased in placental tissues from women with early or late onset PE compared with in the control group (Fig. 1A). No significant difference was detected in FAM99A expression between placental tissues from patients with early onset and late onset PE (Fig. 1A). 

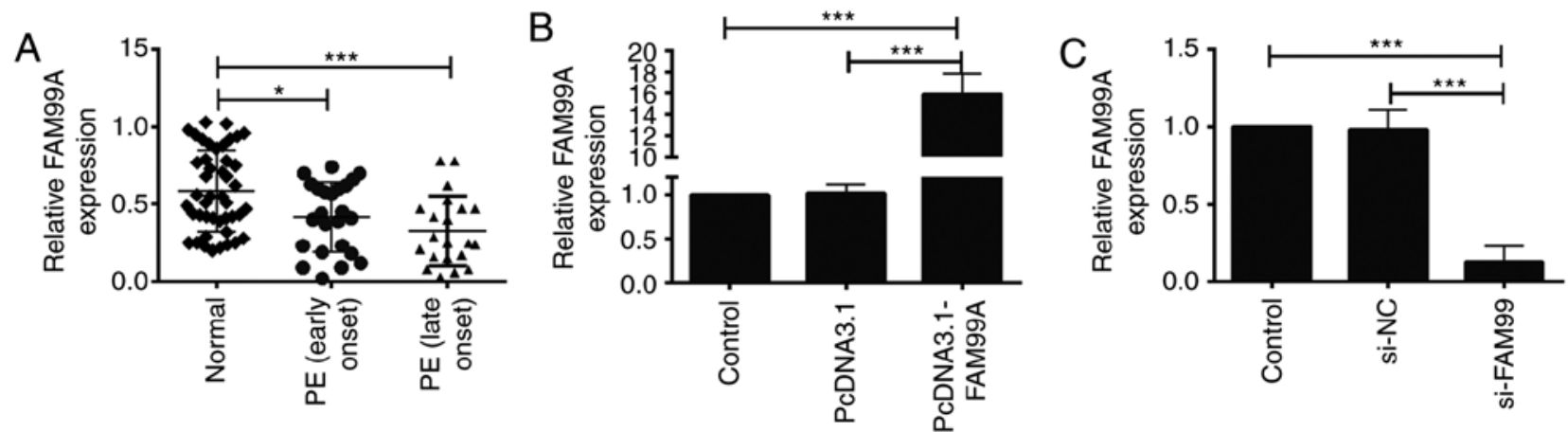

Figure 1. (A) Long non-coding RNA FAM99A was downregulated in placental tissues from patients with PE. FAM99A expression was assessed by RT-qPCR FAM99A expression levels were lower in placental tissues from patients with PE (early onset: $n=24$; late onset: $n=21$ ) compared with in normal placental tissue ( $\mathrm{n}=45$ ). (B) Relative expression levels of FAM99A in HTR-8/SVneo cells were increased post-transfection with pcDNA3.1-FAM99A, as measured by RT-qPCR . (C) Relative expression levels of FAM99A were decreased in HTR-8/SVneo cells post-transfection with si-FAM99A, as measured by RT-qPCR. "P<0.05 and ${ }_{* * * *} \mathrm{P}<0.001$. FAM99A, family with sequence similarity 99 member A; NC, negative control; PE, preeclampsia; RT-qPCR, reverse transcription-quantitative PCR; si, small interfering RNA.
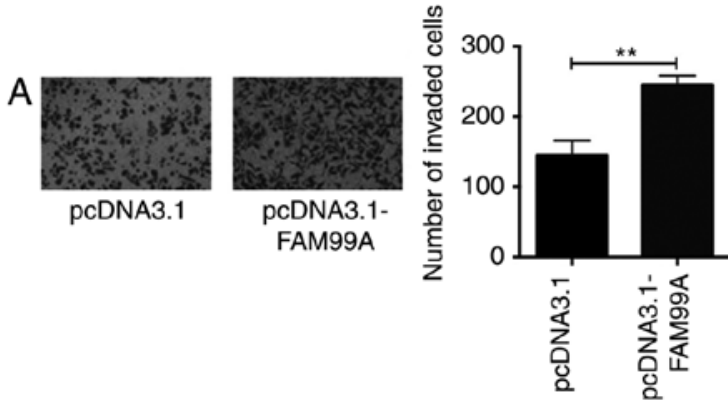

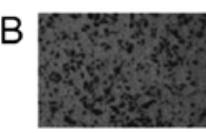

si-NC

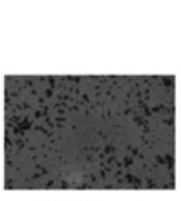

si-FAM99A
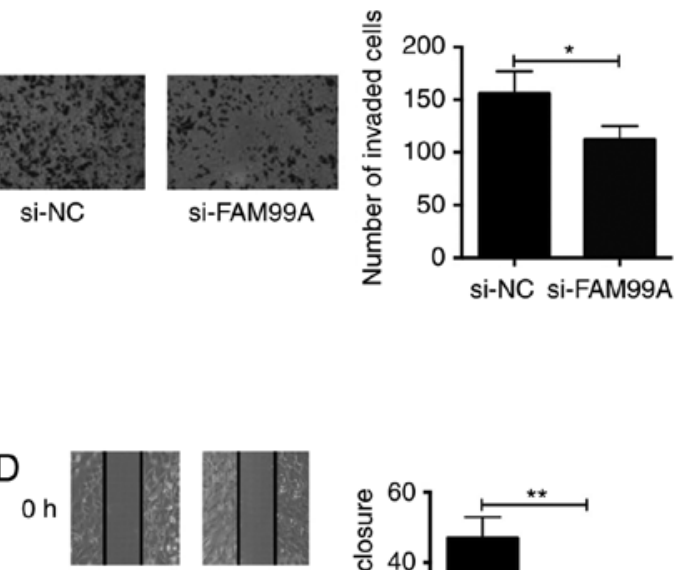

$24 \mathrm{~h}$

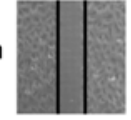

si-NC
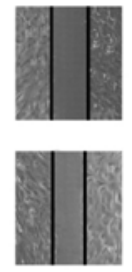

si-FAM99A

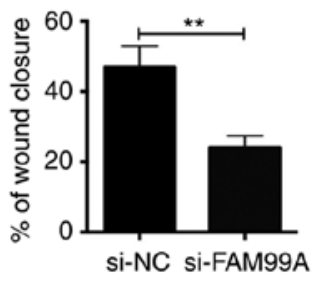

FAM99A

Figure 2. Effects of FAM99A on HTR-8/SVneo cell invasion and migration. Invasive ability of HTR-8/SVneo cells transfected with (A) pcDNA3.1 or pcDNA3.1-FAM99A, and (B) si-NC or si-FAM99A was determined by Transwell invasion assay (magnification, x200). Migration ability of HTR-8/SVneo cells transfected with (C) pcDNA3.1 or pcDNA3.1-FAM99A, and (D) si-NC or si-FAM99A was determined by wound healing assay (magnification, $\mathrm{x} 100$ ). $\mathrm{n}=3$. ${ }^{*} \mathrm{P}<0.05$ and ${ }^{* *} \mathrm{P}<0.01$. FAM99A, family with sequence similarity 99 member A; NC, negative control; si, small interfering RNA.

Effects of FAM99A on the proliferation and migration of HTR-8/SVneo cells. The significant downregulation of FAM99A in placental tissues from women with severe PE suggested a possible role for FAM99A in PE. To examine the potential function of FAM99A in vitro, HTR-8/SVneo trophoblast cells were used in the present study. Transfection with pcDNA3.1-FAM99A increased the expression levels of FAM99A in HTR-8/SVneo cells compared with in cells transfected with pcDNA3.1 (Fig. 1B). si-FAM99A transfection significantly suppressed the expression levels of FAM99A in HTR-8/SVneo cells compared with transfection with si-NC (Fig. 1C). In vitro functional assays, including cell invasion and wound healing assays, were performed to evaluate the invasive and migratory abilities of HTR-8/SVneo cells.
The cell invasion assay revealed that FAM99A overexpression promoted invasion of HTR-8/SVneo cells (Fig. 2A), whereas knockdown of FAM99A suppressed invasion of HTR-8/SVneo cells (Fig. 2B). In addition, the wound healing assay demonstrated that overexpression of FAM99A accelerated wound closure (Fig. 2C); however, knockdown of FAM99A suppressed wound closure in HTR-8/SVneo cells (Fig. 2D).

Effects of FAM99A on HTR-8/SVneo cell apoptosis. To assess the effects of FAM99A on HTR-8/SVneo cell apoptosis, flow cytometry was performed to measure cell apoptotic rate. The results revealed that overexpression of FAM99A decreased the apoptotic rate of cells (Fig. 3A), whereas knockdown 

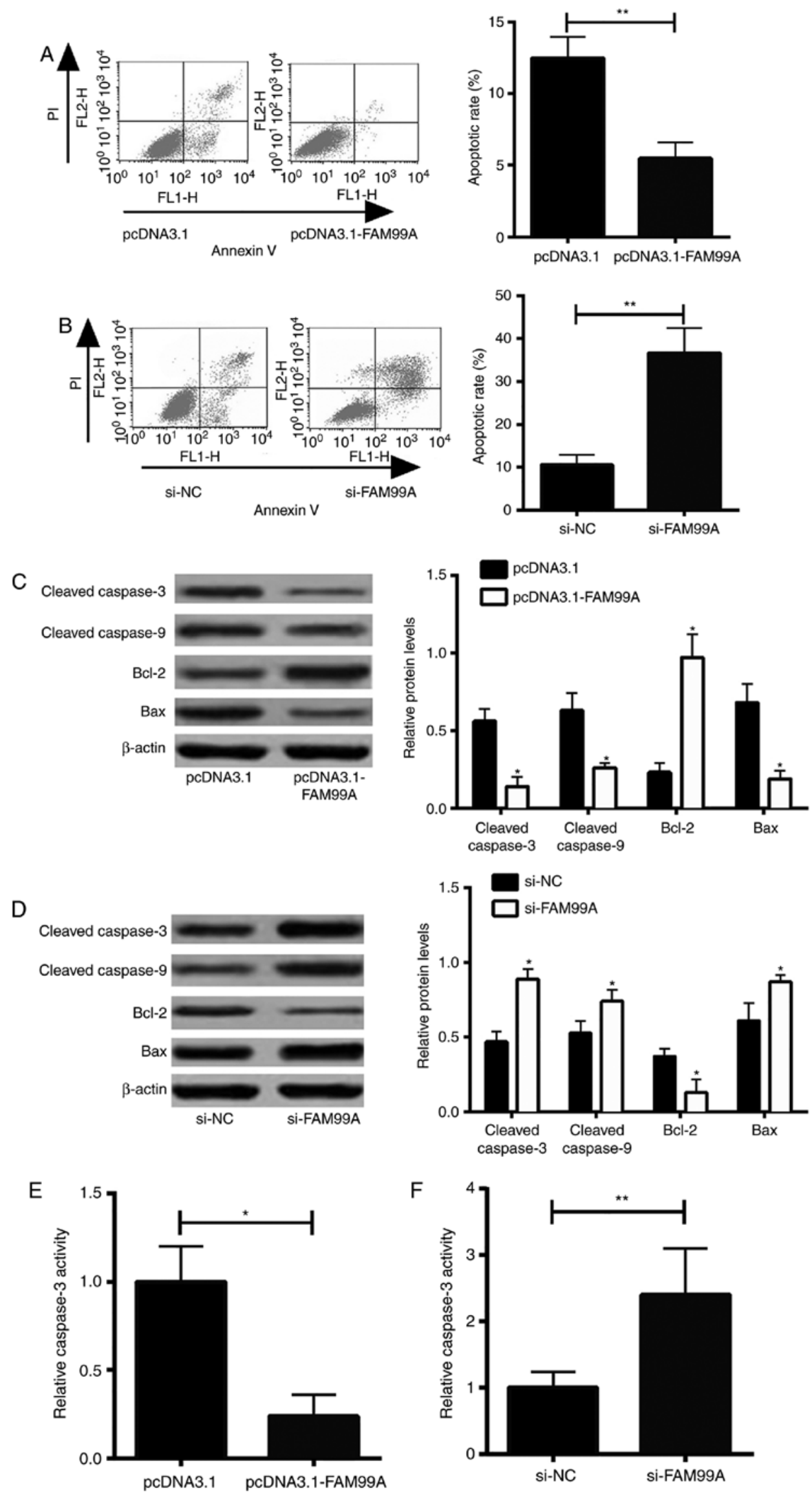

Figure 3. Effects of FAM99A on HTR-8/SVneo cell apoptosis. Apoptotic rate of HTR-8/SVneo cells transfected with (A) pcDNA3.1 or pcDNA3.1-FAM99A, and (B) si-NC or si-FAM99A-transfected HTR-8/SVneo cells was determined by flow cytometry. Cleaved caspase-3, caspase-9, Bcl-2 and Bax protein expression in HTR-8/SVneo cells transfected with (C) pcDNA3.1 or pcDNA3.1-FAM99A, and (D) si-NC or si-FAM99A was determined by western blot analysis. Caspase-3 activity of HTR-8/SVneo cells transfected with (E) pcDNA3.1 or pcDNA3.1-FAM99A, and (F) si-NC or si-FAM99A was determined using a caspase-3 activity assay kit. $\mathrm{n}=3{ }^{*} \mathrm{P}<0.05,{ }^{* *} \mathrm{P}<0.01$ vs. controls. FAM99A, family with sequence similarity 99 member A; NC, negative control; PI, propidium iodide; si, small interfering RNA. 

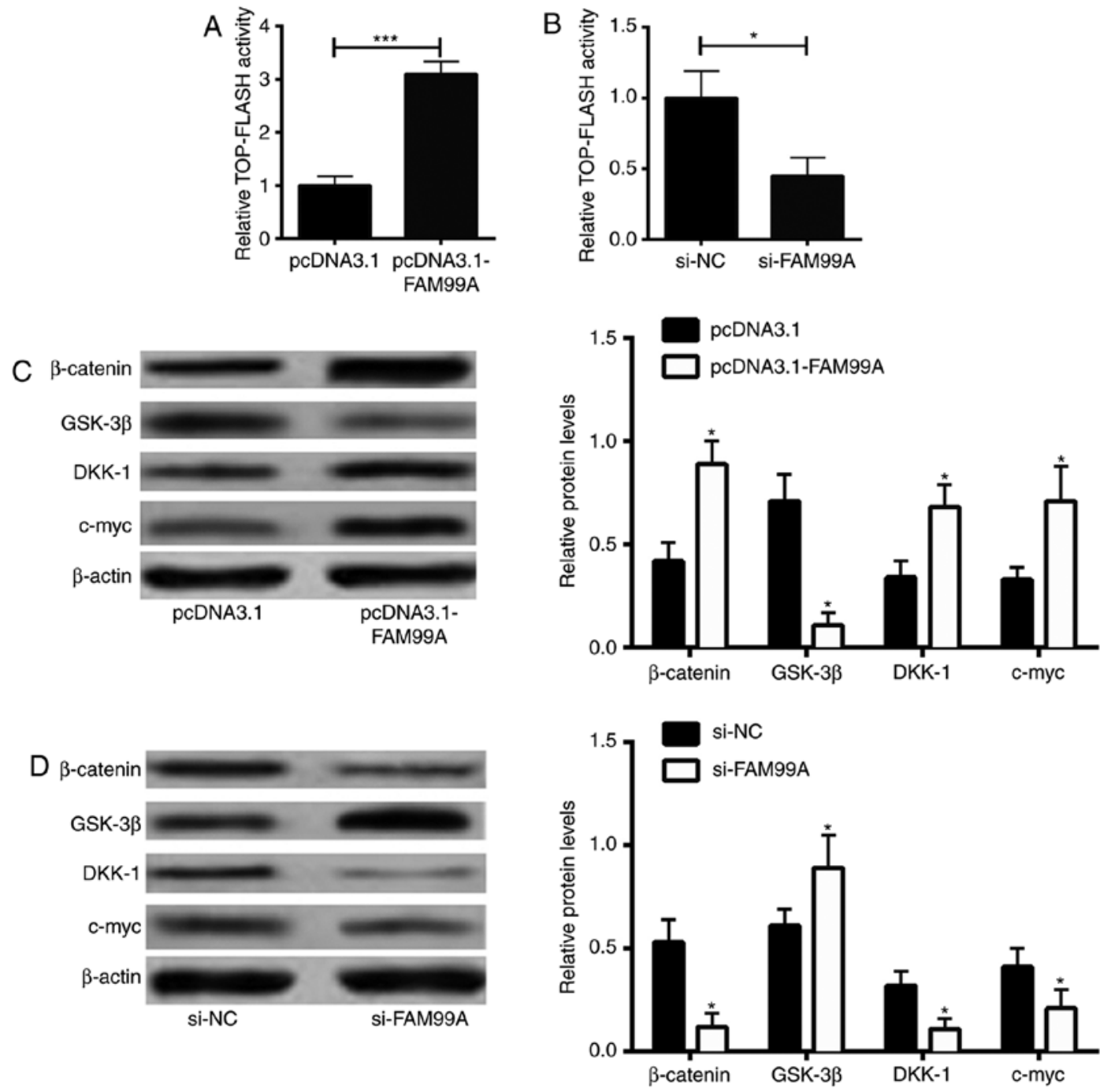

Figure 4. Effects of FAM99A on Wnt/ $\beta$-catenin signaling in HTR-8/SVneo cells. Wnt/ $\beta$-catenin signaling activity of HTR-8/SVneo cells transfected with (A) pcDNA3.1 or pcDNA3.1-FAM99A, and (B) si-NC or si-FAM99A was determined using the TOP-FLASH luciferase assay. $\beta$-catenin, GSK-3 $\beta$, DKK-1 and c-myc protein expression levels in (C) pcDNA3.1- or pcDNA3.1-FAM99A-transfected, and (D) si-NC- or si-FAM99A-transfected HTR-8/SVneo cells were determined by western blot analysis. $\mathrm{n}=3$. ${ }^{*} \mathrm{P}<0.05$ and ${ }^{* * *} \mathrm{P}<0.001$ vs. controls. DKK-1, dickkopf WNT signaling pathway inhibitor 1 ; FAM99A, family with

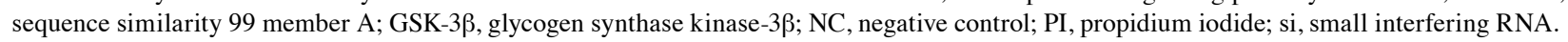

of FAM99A increased the apoptotic rate of HTR-8/SVneo cells (Fig. 3B). In addition, the effects of FAM99A on cell apoptosis-associated protein expression were determined by western blot analysis. The results demonstrated that FAM99A overexpression decreased cleaved caspase-3, cleaved caspase- 9 and Bax protein expression, and increased $\mathrm{Bcl}-2$ protein expression in HTR-8/SVneo cells (Fig. 3C). Knockdown of FAM99A had the opposite effects on cleaved caspase-3, cleaved caspase-9, Bcl-2 and Bax protein expression in HTR-8/SVneo cells (Fig. 3D). Furthermore, overexpression of FAM99A decreased caspase-3 activity in HTR-8/SVneo cells (Fig. 3E), whereas knockdown of FAM99A increased caspase-3 activity in HTR-8/SVneo cells (Fig. 3F).

Effects of FAM99A on Wnt/ $\beta$-catenin signaling in HTR-8/SVneo cells. To assess the role of FAM99A in Wnt/ $\beta$-catenin signaling activities, a TOP-FLASH luciferase assay was performed. Overexpression of FAM99A increased TOP-FLASH activity (Fig. 4A), whereas knockdown of FAM99A suppressed TOP-FLASH activity in HTR-8/SVneo cells (Fig. 4B), suggesting the enhanced effects of FAM99A on the Wnt/ $\beta$-catenin signaling activity. In addition, $\beta$-catenin, glycogen synthase kinase (GSK)-3 $\beta$, DKK-1 and c-myc protein expression levels were determined by western blot analysis. As shown in Fig. 4C, overexpression of FAM99A increased the protein expression levels of $\beta$-catenin, dickkopf WNT signaling pathway inhibitor 1 (DKK-1) and c-myc, and decreased the protein expression levels of GSK-3 $\beta$ in HTR-8/SVneo cells. Conversely, knockdown of FAM99A decreased the protein expression levels of $\beta$-catenin, DKK-1 and c-myc, and upregulated GSK-3 $\beta$ protein expression (Fig. 4D).

\section{Discussion}

Recently, accumulating evidence has indicated that lncRNAs may serve essential roles in PE development and progression (19). The present findings revealed that dysregulation of FAM99A could affect trophoblast cell invasion, migration and apoptosis, suggesting a potential role for FAM99A in the occurrence and development of PE. 
Previous studies have identified associations between lncRNAs and the pathogenesis of PE. Among these lncRNAs, SPRY4-IT1, Uc.187 and replication protein A-interacting protein are upregulated in placental tissues from women with severe PE, and these lncRNAs serve suppressive roles in trophoblast cell invasion and migration $(13,20,21)$. By contrast, downregulated lncRNAs (MEG3, MALAT1, H19, ATB, taurine up-regulated 1 and plasmacytoma variant translocation 1) in placental tissues from women with PE have been identified to enhance trophoblast cell invasion and migration $(11,12,22-25)$. To date, little is known regarding the role of FAM99A in the pathogenesis of PE. A previous study reported that FAM99A rs7131362 is associated with maternal circulating clinically relevant triglyceride concentrations early in pregnancy (26). Petry et al (27) demonstrated that fetal FAM99A rs1489945 is associated with maternal mean arterial blood pressure. Microarray analysis also indicated that FAM99A is downregulated in the placental tissues from patients with PE (14). In the present study, FAM99A was significantly downregulated in the placental tissues from women with PE compared with in those from healthy pregnancies, thus suggesting that a decreased level of FAM99A may be associated with the progression and development of PE. Previous studies have identified that impairment of spiral artery remodeling contributes to the pathogenesis of PE (28), and impaired EVTs serve a key role in the pathogenesis of PE (29). In the present study, in vitro functional assays revealed that overexpression of FAM99A promoted trophoblast cell invasion and migration, and inhibited apoptosis, whereas downregulation of FAM99A suppressed trophoblast cell invasion and migration, and induced apoptosis. These results suggested that downregulation of FAM99A in the placental tissues may exert a suppressive effect on trophoblasts.

The Wnt/ $\beta$-catenin pathway functions to modulate essential biological processes, such as cell invasion, migration, apoptosis and proliferation, and it belongs to the canonical Wnt-signaling pathway (30). Dysregulation of the Wnt/ $\beta$-catenin signaling pathway has been reported to serve a key role in various types of human disease, particularly the development of human cancer $(31,32)$. Previous evidence has indicated that abnormal activation of $\mathrm{Wnt} / \beta$-catenin signaling may be associated with the pathogenesis of PE. Zhuang et al (33) reported that the staining intensity of $\beta$-catenin is decreased in placental tissues from women with PE. In addition, oxidative stress-induced C/EBP $\beta$ inhibits the activities of $\mathrm{Wnt} / \beta$-catenin signaling, which subsequently contributes to the pathogenesis of PE (34). A study using different activators of $\mathrm{Wnt} / \beta$-catenin signaling revealed that $\mathrm{Wnt} / \beta$-catenin signaling is closely related to trophoblast cell differentiation (35). In addition, decreased expression of WNT2 in the villi of patients with unexplained recurrent spontaneous abortion may cause trophoblast cell dysfunction via suppressing Wnt/ $\beta$-catenin signaling (36). On this basis, the present study further examined the effects of FAM99A on Wnt/ $\beta$-catenin signaling activity. The results demonstrated that overexpression of FAM99A increased Wnt/ $\beta$-catenin signaling activity, whereas knockdown of FAM99A decreased Wnt/ $\beta$-catenin signaling activity, suggesting that the effects of FAM99A on the biological behaviors of trophoblasts may involve modulation of Wnt $/ \beta$-catenin signaling.

The present study has several limitations. Firstly, the expression of FAM99A in the human placenta throughout gestation was not known. Future studies in which the expression profile of FAM99A in the peripheral blood from patients with PE is monitored throughout gestation are required to confirm the role of FAM99A in the pathogenesis of PE. Secondly, the timing of caesarean section varied among the patients and this may have contributed to the differential expression of FAM99A. Thirdly, as various factors may affect the expression of FAM99A in clinical samples, such as fetal distress and pre-labor rupture of membranes, caution should be applied in interpreting the results. Future studies may investigate these factors in order to verify the present findings regarding the role of FAM99A in PE.

In conclusion, the present results indicated that the lncRNA FAM99A was downregulated in placental tissues from patients with PE. Downregulation of FAM99A may lead to suppressed cell invasion and migration, and increased cell apoptosis in trophoblasts, which may impair the process of spiral artery remodeling. The biological effects of FAM99A on trophoblasts may involve modulation of $\mathrm{Wnt} / \beta$-catenin signaling.

\section{Acknowledgements}

The authors would like to thank Dr L. Zhang (Department of Biomedical Sciences, Xi'an Medical University) for his support in performing statistical analysis.

\section{Funding}

The present study was supported by The Northwest Women and Children's Hospital.

\section{Availability of data and materials}

The datasets used during the present study are available from the corresponding author upon reasonable request.

\section{Authors' contributions}

YC designed the study. TH, YQ and YL performed the experiments, analyzed the data and wrote the manuscript. JW and $\mathrm{RH}$ performed the statistical analysis. All authors read and approved the final manuscript.

\section{Ethics approval and consent to participate}

All experiments were approved by the Ethics Committee of The Northwest Women and Children's Hospital, and written informed consent was obtained from all the recruited subjects.

\section{Patient consent for publication}

Not applicable.

\section{Competing interests}

The authors declare that they have no competing interests. 


\section{References}

1. Kurtz WS, Glueck CJ, Hutchins RK, Sisk RA and Wang P: Retinal artery and vein thrombotic occlusion during pregnancy: Markers for familial thrombophilia and adverse pregnancy outcomes. Clin Ophthalmol 10: 935-938, 2016.

2. Powe CE, Levine RJ and Karumanchi SA: Preeclampsia, a disease of the maternal endothelium: The role of antiangiogenic factors and implications for later cardiovascular disease. Circulation 123: 2856-2869, 2011

3. Gao L, Qi HB, Kamana KC, Zhang XM, Zhang H and Baker PN: Excessive autophagy induces the failure of trophoblast invasion and vasculature: Possible relevance to the pathogenesis of preeclampsia. J Hypertens 33: 106-117, 2015.

4. Salomon C, Yee SW, Mitchell MD and Rice GE: The possible role of extravillous trophoblast-derived exosomes on the uterine spiral arterial remodeling under both normal and pathological conditions. Biomed Res Int 2014: 693157, 2014.

5. Saito S and Nakashima A: A review of the mechanism for poor placentation in early-onset preeclampsia: The role of autophagy in trophoblast invasion and vascular remodeling. J Reprod Immunol 101-102: 80-88, 2014.

6. Hung TH, Chen SF, Lo LM, Li MJ, Yeh YL and Hsieh TT: Increased autophagy in placentas of intrauterine growth-restricted pregnancies. PLoS One 7: e40957, 2012.

7. Sharp AN, Heazell AE, Crocker IP and Mor G: Placental apoptosis in health and disease. Am J Reprod Immunol 64: 159-169, 2010.

8. Wieczorek E and Reszka E: mRNA, microRNA and lncRNA as novel bladder tumor markers. Clin Chim Acta 477: 141-153, 2018

9. Freedman JE and Miano JM and National Heart, Lung, and Blood Institute Workshop Participants: Challenges and opportunities in linking long noncoding RNAs to cardiovascular, lung, and blood diseases. Arterioscler Thromb Vasc Biol 37: 21-25, 2017.

10. Tian L, Zheng F, Li Z, Wang H, Yuan H, Zhang X, Ma Z, Li X, Gao $X$ and Wang B: miR-148a-3p regulates adipocyte and osteoblast differentiation by targeting lysine-specific demethylase $6 \mathrm{~b}$. Gene 627: 32-39, 2017.

11. Zhang Y, Zou Y, Wang W, Zuo Q, Jiang Z, Sun M, De W and Sun L: Down-regulated long non-coding RNA MEG3 and its effect on promoting apoptosis and suppressing migration of trophoblast cells. J Cell Biochem 116: 542-550, 2015.

12. Chen H, Meng T, Liu X, Sun M, Tong C, Liu J, Wang H and Du J: Long non-coding RNA MALAT-1 is downregulated in preeclampsia and regulates proliferation, apoptosis, migration and invasion of JEG-3 trophoblast cells. Int J Clin Exp Pathol 8: 12718-12727, 2015.

13. Zuo Q, Huang S, Zou Y, Xu Y, Jiang Z, Zou S, Xu H and Sun L: The Lnc RNA SPRY4-IT1 modulates trophoblast cell invasion and migration by affecting the epithelial-mesenchymal transition. Sci Rep 6: 37183, 2016.

14. He X, He Y, Xi B, Zheng J, Zeng X, Cai Q, Ouyang Y, Wang C, Zhou $\mathrm{X}$, Huang $\mathrm{H}$, et al: LncRNAs expression in preeclampsia placenta reveals the potential role of LncRNAs contributing to preeclampsia pathogenesis. PLoS One 8: e81437, 2013.

15. Peng L, Liu Z, Xiao J, Tu Y, Wan Z, Xiong H, Li Y and Xiao W: MicroRNA-148a suppresses epithelial-mesenchymal transition and invasion of pancreatic cancer cells by targeting Wnt10b and inhibiting the Wnt/ $\beta$-catenin signaling pathway. Oncol Rep 38 : 301-308, 2017

16. Tranquilli AL, Brown MA, Zeeman GG, Dekker G and Sibai BM: The definition of severe and early-onset preeclampsia. Statements from the international society for the study of hypertension in pregnancy (ISSHP). Pregnancy Hypertens 3: 44-47, 2013.

17. Graham CH,Hawley TS, Hawley RG, MacDougall JR, Kerbel RS, Khoo N and Lala PK: Establishment and characterization of first trimester human trophoblast cells with extended lifespan. Exp Cell Res 206: 204-211, 1993.

18. Livak KJ and Schmittgen TD: Analysis of relative gene expression data using real-time quantitative PCR and the 2(-Delta Delta C(T)) method. Methods 25: 402-408, 2001.
19. McAninch D, Roberts CT and Bianco-Miotto T: Mechanistic insight into long noncoding RNAs and the placenta. Int J Mol Sci 18: E1371, 2017.

20. Zou Y, Jiang Z, Yu X, Sun M, Zhang Y, Zuo Q, Zhou J, Yang N, Han P, Ge Z, et al: Upregulation of long noncoding RNA SPRY4-IT1 modulates proliferation, migration, apoptosis, and network formation in trophoblast cells HTR-8SV/neo. PLoS One 8: e79598, 2013

21. Song X, Rui C, Meng L, Zhang R, Shen R, Ding H, Li J, Li J and Long W: Long non-coding RNA RPAIN regulates the invasion and apoptosis of trophoblast cell lines via complement protein Clq. Oncotarget 8: 7637-7646, 2017.

22. Zuckerwise L, Li J, Lu L, Men Y, Geng T, Buhimschi CS, Buhimschi IA, Bukowski R, Guller S, Paidas M and Huang Y: H19 long noncoding RNA alters trophoblast cell migration and invasion by regulating T $\beta \mathrm{R} 3$ in placentae with fetal growth restriction. Oncotarget 7: 38398-38407, 2016.

23. Liu X, Chen H, Kong W, Zhang Y, Cao L, Gao L and Zhou R: Down-regulated long non-coding RNA-ATB in preeclampsia and its effect on suppressing migration, proliferation, and tube formation of trophoblast cells. Placenta 49: 80-87, 2017.

24. Xu Y, Ge Z, Zhang E, Zuo Q, Huang S, Yang N, Wu D, Zhang Y, Chen Y, Xu H, et al: The lncRNA TUG1 modulates proliferation in trophoblast cells via epigenetic suppression of RND3. Cell Death Dis 8: e3104, 2017.

25. Xu H, Sun Q, Lu L, Luo F, Zhou L, Liu J, Cao L, Wang Q, Xue J, Yang Q, et al: MicroRNA-218 acts by repressing TNFR1-mediated activation of $\mathrm{NF}-\kappa \mathrm{B}$, which is involved in MUC5AC hyper-production and inflammation in smoking-induced bronchiolitis of COPD. Toxicol Lett 280: 171-180, 2017.

26. Petry CJ, Koulman A, Lu L, Jenkins B, Furse S, Prentice P, Matthews L, Hughes IA, Acerini CL, Ong KK and Dunger DB: Associations between the maternal circulating lipid profile in pregnancy and fetal imprinted gene alleles: A cohort study. Reprod Biol Endocrinol 16: 82, 2018.

27. Petry CJ, Sanz Marcos N, Pimentel G, Hayes MG, Nodzenski M, Scholtens DM, Hughes IA, Acerini CL, Ong KK, Lowe WL Jr and Dunger DB: Associations between fetal imprinted genes and maternal blood pressure in pregnancy. Hypertension 68 : 1459-1466, 2016

28. Freitag N, Tirado-González I, Barrientos G, Herse F, Thijssen VL, Weedon-Fekjær SM, Schulz H, Wallukat G, Klapp BF, Nevers T, et al: Interfering with Gal-1-mediated angiogenesis contributes to the pathogenesis of preeclampsia. Proc Natl Acad Sci USA 110: 11451-11456, 2013.

29. Palei AC, Spradley FT, Warrington JP, George EM and Granger JP: Pathophysiology of hypertension in pre-eclampsia: A lesson in integrative physiology. Acta Physiol (Oxf) 208: 224-233, 2013.

30. Hoppler S and Kavanagh CL: Wnt signalling: Variety at the core. J Cell Sci 120: 385-393, 2007.

31. Krishnamurthy N and Kurzrock R: Targeting the Wnt/beta-catenin pathway in cancer: Update on effectors and inhibitors. Cancer Treat Rev 62: 50-60, 2017.

32. Nusse R and Clevers $H$ : Wnt/ $/$-catenin signaling, disease, and emerging therapeutic modalities. Cell 169: 985-999, 2017.

33. Zhuang B, Luo X, Rao H, Li Q, Liu X and Qi H: Expression and significance of SATB1 and wnt/ $\beta$-catenin signaling molecule in the placenta of preeclampsia. Zhonghua Fu Chan Ke Za Zhi 50: 283-290, 2015 (In Chinese).

34. Zhuang B, Luo X, Rao H, Li Q, Shan N, Liu X and Qi H: Oxidative stress-induced C/EBP $\beta$ inhibits $\beta$-catenin signaling molecule involving in the pathology of preeclampsia. Placenta 36 : 839-846, 2015.

35. Kumar P, Thirkill TL, Ji J, Monte LH and Douglas GC: Differential effects of sodium butyrate and lithium chloride on rhesus monkey trophoblast differentiation. PLoS One 10: $\mathrm{e} 0135089,2015$.

36. Yang Y, Wang Y, Liang Q, Yao L, Gu S and Bai X: MiR-338-5p promotes inflammatory response of fibroblast-like synoviocytes in rheumatoid arthritis via targeting SPRY1. J Cell Biochem 118: 2295-2301, 2017. 